Écrire

l'histoire

\section{Écrire l'histoire}

Histoire, Littérature, Esthétique

\section{9 | 2019}

\section{L'historien et les langues}

\title{
La Babel des sciences
}

Michael D. GoRDIN, Scientific Babel. The language of science from the fall of Latin to the rise of English (2017)

\section{Sophie Cœuré}

\section{(2) OpenEdition}

Journals

Édition électronique

URL : http://journals.openedition.org/elh/2153

DOI : 10.4000/elh.2153

ISSN : 2492-7457

Éditeur

CNRS Éditions

Édition imprimée

Date de publication : 1 décembre 2019

Pagination : 200-203

ISBN : 978-2-271-12967-3

ISSN : 1967-7499

Référence électronique

Sophie Cœuré, «La Babel des sciences », Écrire l'histoire [En ligne], 19 | 2019, mis en ligne le 01 décembre 2019, consulté le 16 février 2021. URL : http://journals.openedition.org/elh/2153 ; DOI : https://doi.org/10.4000/elh.2153

Ce document a été généré automatiquement le 16 février 2021.

Tous droits réservés 


\title{
La Babel des sciences
}

\author{
Michael D. GORDIN, Scientific Babel. The language of science from the fall \\ of Latin to the rise of English (2017)
}

\section{Sophie Cœuré}

\section{RÉFÉRENCE}

Michael D. GORDIN, Scientific Babel. The language of science from the fall of Latin to the rise of English, Londres, Profile Books, 2017, 415 p.

1 Professeur d'histoire des sciences à l'université de Princeton, Michael Gordin propose un bel essai sur la manière dont on "parle les sciences » ("Talking science »). À partir du constat bien partagé du triomphe de l'anglais global, il repart du latin comme "paradis perdu», s'appuyant sur cette magnifique et pessimiste réflexion de d'Alembert dans le Discours préliminaire de l'Encyclopédie en 1751 : «Ainsi, avant la fin du XVIII siècle, un philosophe qui voudra s'instruire à fond des découvertes de ses prédécesseurs, sera contraint de charger sa mémoire de sept à huit langues différentes ; et après avoir consumé à les apprendre le temps le plus précieux de sa vie, il mourra avant de commencer à s'instruire. » Prenant en compte les sciences « exactes », sans la médecine ni les sciences humaines et sociales, l'auteur s'attache à historiciser la «profusion limitée» des grandes langues véhiculaires savantes (soit par ordre alphabétique français : allemand, anglais, arabe, chinois classique, danois, français, grec ancien, italien, japonais, latin, néerlandais, persan, russe, sanskrit, suédois, syriaque, turc ottoman) et l'évolution vers le monolinguisme. Comment cette évolution a-t-elle été prise en compte par la réflexion sur le métalangage scientifique et les utopies de langue universelle?

2 Michael Gordin s'appuie sur ses recherches personnelles - notamment dans les chapitres passionnants consacrés aux nomenclatures chimiques. Il mobilise aussi de vastes lectures, toujours citées en langue originale, car, écrit-il fort justement, « the past did not happen exclusively in English, though many histories make it seem as though it did ». Il est tout à fait conscient des biais de sources qui pèsent sur son propre travail (il ne lit 
pas les langues asiatiques, et centre de fait son analyse sur le monde occidental), mais aussi sur les tableaux statistiques d'usage des langues, élaborés à partir des « abstract journals »; or les savants citent de préférence leur propre langue.

3 Pour commencer, l'exemple du latin permet d'insister sur les principaux attendus d'une langue scientifique: il faut qu'elle soit flexible, pratiquée, désirable comme universelle. La combinaison de ces facteurs contribue à expliquer l'ignorance du latin sur le sous-continent indien, où s'imposa le sanskrit, son blocage en Chine, puis, pour d'autres raisons, son déclin dans l'Europe de la Réforme. Ainsi, souligne l'auteur, si le latin supplanta le grec et l'arabe comme langue de science, il ne fut jamais une langue globale.

4 L'étude de cas des tables de nomenclatures chimiques internationales permet ensuite à Michael Gordin d'insister sur la montée en puissance de l'allemand comme langue scientifique au $\mathrm{XIX}^{\mathrm{e}}$ siècle, mais aussi sur une première légitimation du russe, malgré l'échec de la tentative assez fascinante, vers 1870, d'utiliser la forme russe «nom / patronyme» pour nommer les éléments chimiques (ce qui donnait par exemple " potassimum chlorovich »). Avant 1914, l'évolution vers une simplification progressive des sciences en Europe et en Amérique du Nord vers trois langues - le français, qui est également la langue de la diplomatie, l'allemand et l'anglais - s'accompagne d'une crainte de la "babélisation", entraînant la recherche d'une langue internationale souple et fonctionnelle. La version la plus aboutie en sera l'ido - une dérivation de l'espéranto. L'un de ses promoteurs, Wilhelm Ostwald, né à Riga, Prix Nobel de chimie en 1909, fonde en 1911 l'organisation internationale Brücke dédiée à la standardisation des sciences, avant d'élaborer, pendant le premier conflit mondial, le plan d'un allemand simplifié qui redonnerait force et vigueur à sa langue et permettrait un triomphe de la "civilisation». Cette mobilisation des savants par les nationalismes affrontés pendant la guerre, bien étudiée en France, notamment par Anne Rasmussen, fait du politique le facteur d'explication dominant de l'évolution de l'entre-deuxguerres. Gordin montre bien l'impact à long terme du boycott de l'allemand dans les sciences internationales dans les années 1920, mais aussi, ce qui est moins connu, les effets de l'épuration par les nazis des milieux scientifiques juifs - largement exilés aux États-Unis - et de la langue elle-même.

5 La guerre froide est de nouveau analysée au prisme de la politisation des sciences. D'un côté, l'Union soviétique tend vers l'autarcie - tout en soignant l'apprentissage de l'anglais par ses élites scientifiques; le russe devient "langue de classe ", donc à visée universelle. De l'autre, les États-Unis mettent en œuvre précocement des parades à la montée en puissance du russe, par l'enseignement et par la traduction. La question des langues ouvre vers des enjeux bien plus larges : ainsi la mise en œuvre des programmes de "machine translation", nés de l'impératif de traduction massive et standardisée, aura-t-elle des conséquences majeures sur le développement de l'informatique aux États-Unis. De même, la traduction, humaine cette fois, des articles scientifiques en russe, pratiquée à large échelle dans des publications dédiées et financées par les pouvoirs publics américains, aura-t-elle un impact paradoxal sur le développement de l'anglais, qui devient le point d'entrée mondial pour accéder à la science soviétique. Les nouvelles tentatives pour créer une communauté textuelle universelle qui pallierait l'absence de contacts Est-Ouest - par l'espéranto, peu pratiqué dans les milieux scientifiques avant la Seconde Guerre mondiale ; par la création de l'interlingua, langue uniquement écrite - sont un échec. L'anglais s'impose d'autant plus aisément que, pour 
des raisons, politiques toujours, sur lesquelles l'auteur peine un peu à s'expliquer, il est perçu comme "neutre » dans un monde en phase de décolonisation, face au français, mais aussi au russe.

6 Michael Gordin conclut son essai en ouvrant des perspectives d'analyse dans deux directions, articulées avec la ligne de force politique de son ouvrage. Une réflexion s'impose tout d'abord à l'évidence sur la langue anglaise, dans sa déclinaison scientifique à l'américaine, comme vecteur d'écriture de la science, désormais monopolistique malgré quelques résistances, notamment chez les mathématiciens français. L'auteur souligne la force de l'anglais - syntaxe simple, capacité à absorber des termes étrangers-, mais aussi ses faiblesses - réduction du vocabulaire, appauvrissement du raisonnement lié à la forme standard "IMRAD» (Introduction, Methods, Results And Discussion + Conclusion) qui structure désormais les articles tant en sciences dures que, de plus en plus, en sciences humaines. Il esquisse aussi une analyse des conséquences profondes de l'écriture scientifique en anglais "global» par des auteurs d'une autre langue maternelle, reçue de même par des lecteurs qui ignorent largement les conditions politiques, sociales et matérielles de production de ces recherches. Cela interroge alors rétrospectivement sur un point non abordé par l'auteur dans les chapitres précédents: quelle fut la pratique des langues dans les contacts scientifiques de guerre froide, notamment dans les congrès internationaux, avec quel impact sur la perception d'une "communauté scientifique " transnationale et, en retour, sur les politiques et les pratiques scientifiques nationales? La seconde piste proposée par Gordin renvoie à la recherche de la langue universelle, au prisme des projets de communication de la connaissance avec les univers extraterrestres, par exemple via la lincos (lingua cosmica), élaborée par le mathématicien Hans Freudenthal, qui utilise les ondes radio. La science est-elle en elle-même un langage, dégagé de l'historicité politique, sociale, voire humaine, que son universalité amènerait à supplanter tous les autres? Question de philosophe autant que d'historien, à laquelle Michael Gordin se garde bien de répondre.

\section{INDEX}

oeuvrecitee Scientific Babel. The language of science from the fall of Latin to the rise of English (Michael D. Gordin, 2017)

\section{AUTEURS}

\section{SOPHIE CEEURÉ}

Sophie Cœuré est professeure d'histoire contemporaine à l'université Paris Diderot - Université de Paris. S'appuyant pour la première fois sur des archives tant russes que françaises, elle a proposé une analyse pionnière des engagements et des mythologies communistes et soviétiques avec notamment "Cousu de fil rouge ». Voyages des intellectuels français en Union soviétique (dirigé avec Rachel Mazuy, CNRS Éd., 2012), Pierre Pascal. La Russie entre christianisme et communisme (Noir 
sur Blanc, 2014), La Grande Lueur à l'Est. Les Français et l'Union soviétique (1917-1939) (CNRS Éd., 2017).

Elle a contribué au renouveau des recherches sur l'histoire politique des archives et des spoliations culturelles dans La Mémoire spoliée. Les archives des Français, butin de guerre nazi puis soviétique, de 1940 à nos jours (Payot \& Rivages, 2017). L'histoire est aussi un jeu du destin, et Sophie Cœuré a participé à l'uchronie L'Autre Siècle (Fayard, 2018), en imaginant une Russie où la révolution de 1917 n'aurait pas eu lieu. 